\title{
Expanded risk groups help determine which prostate radiotherapy sub-group may benefit from adjuvant androgen deprivation therapy Matthew Beasley ${ }^{1}$, Scott G Williams ${ }^{2}$, Tom Pickles*1 ${ }^{*}$ and The BCCA Prostate Outcomes Unit ${ }^{1}$
}

Address: ${ }^{1}$ British Columbia Cancer Agency, Vancouver, Canada and ${ }^{2}$ Peter MacCallum Cancer Centre, Melbourne, Australia

Email: Matthew Beasley - mbeasley@doctors.org.uk; Scott G Williams - Scott.Williams@petermac.org; Tom Pickles* - tpickles@bccancer.bc.ca; The BCCA Prostate Outcomes Unit - PCOI@bccancer.bc.ca

* Corresponding author

Published: 18 April 2008

Radiation Oncology 2008, 3:8 doi:10.1186/1748-7|7X-3-8

This article is available from: http://www.ro-journal.com/content/3/l/8

(c) 2008 Beasley et al; licensee BioMed Central Ltd.

This is an Open Access article distributed under the terms of the Creative Commons Attribution License (http://creativecommons.org/licenses/by/2.0), which permits unrestricted use, distribution, and reproduction in any medium, provided the original work is properly cited.

\begin{abstract}
Purpose: To assess whether an expanded (five level) risk stratification system can be used to identify the sub-group of intermediate risk patients with prostate cancer who benefit from combining androgen deprivation therapy (ADT) with external beam radiotherapy (EBRT).
\end{abstract}

Materials and methods: Using a previously validated 5-risk group schema, a prospective nonrandomized data set of I423 men treated at the British Columbia Cancer Agency was assessed for the primary end point of biochemical control (bNED) with the RTOG-ASTRO "Phoenix" definition (lowest PSA to date $+2 \mathrm{ng} / \mathrm{mL}$ ), both with and without adjuvant ADT. The median follow-up was 5 years.

Results: There was no bNED benefit for ADT in the low or low intermediate groups but there was a statistically significant bNED benefit in the high intermediate, high and extreme risk groups. The 5 -year bNED rates with and without ADT were $70 \%$ and $73 \%$ respectively for the low intermediate group ( $p=$ non-significant) and $72 \%$ and $58 \%$ respectively for the high intermediate group $(p=0.002)$.

Conclusion: There appears to be no advantage to ADT where the Gleason score is 6 or less and PSA is 15 or less. ADT is beneficial in patients treated to standard dose radiation with Gleason 6 disease and a PSA greater than 15 or where the Gleason score is 7 or higher.

\section{Background}

Androgen deprivation therapy (ADT) has a proven role in the treatment of metastatic prostate cancer. Some groups of patients undergoing external beam radiotherapy (EBRT) for localized prostate cancer also benefit from adjuvant ADT. An EORTC trial randomized patients with T1-2 high grade or T3-4 N0-1 prostate cancer to either radiotherapy alone or with 3 years of ADT and showed an improved overall survival at 5 years[1]. The Trans-Tasman
Radiation Oncology Group 96.01 trial randomized patients with T2b-T4 N0 disease to radiotherapy alone or with 3 or 6 months ADT. There was an improvement in disease free survival for both ADT arms compared to EBRT alone [2].

Sub-division of prostate cancer patients into risk groups can be used to guide management decisions based on their risk of relapse. The National Comprehensive Cancer 
Network (NCCN) classifies three risk groups: Low risk (T1-T2a, Gleason score $\leq 6$ and PSA $\leq 10 \mathrm{ng} / \mathrm{ml}$ ), Intermediate risk (T2b-T2c or Gleason score 7 or PSA 10.1-20 ng/ $\mathrm{ml}$ ) and High risk ( $\geq$ T3a or Gleason $8-10$ or PSA $>20 \mathrm{ng} /$ $\mathrm{ml}$ ) [3]. When localized prostate cancer is divided into three risk groups, an improvement in the biochemical control rate (bNED) at 5 years has been documented in the intermediate and high risk groups by the addition of neo-adjuvant ADT to EBRT [2,4]. However, a high heterogeneity of outcomes within the intermediate risk patient group has been demonstrated [5]. As ADT causes potentially significant morbidity, it would be desirable to identify within the intermediate risk group a lower risk subgroup who can avoid ADT without compromising cancer control. A five-level risk stratification system with expanded intermediate risk divisions has previously been developed using recursive partitioning analysis and externally validated [6] (see Table 1). The aim of the present study is to determine if these five patient subgroups can better identify those who benefit from the combination of ADT with EBRT.

\section{Methods}

A prospective non-randomized patient data set was analyzed, comprising 1583 men treated with EBRT between 1994 and 2001 identified from the Prostate Cohort Outcomes Initiative Database of the British Columbia Cancer Agency (BCCA). After exclusions descried below, 1423 men were available for analysis. All patients received radical EBRT with photon irradiation and CT planning. Those treated with hypofractionated radiation (50-55 Gy in 20 fractions, $\mathrm{n}=133$ ) were excluded, as were those who had neoadjuvant ADT of duration $<2$ months or $>12$ months, $\mathrm{n}=24$. Three-dimensional conformal radiotherapy was used from 1998. The median dose administered was 66 Gy (range 66 - 72 Gy) in 2 Gy fractions. Most patients were treated with small volumes to the prostate alone but 177 patients also had a first phase with whole pelvic radiotherapy. Three patients enrolled in a study of ADT versus ADT and EBRT (National Cancer Institute of Canada PR3 study) were excluded.

Table I: Five Level Risk Stratification for prostate cancer. [6]

\begin{tabular}{lccc}
\hline Risk Group & PSA & $\begin{array}{c}\text { Risk Factor } \\
\text { Gleason }\end{array}$ & T-Stage \\
\hline I. Low & $<7.5$ & $\leq 6$ & any \\
2. Low-intermediate & $7.5-15$ & $\leq 6$ & any \\
3. High-intermediate & $15-20$ & $\leq 6$ & any \\
& $\leq 10$ & $\geq 7$ & any \\
4. High & $20-30$ & $\leq 6$ & any \\
& $10-20$ & $\geq 7$ & any \\
5. Extreme & $>20$ & $\geq 7$ & any \\
& $>30$ & $\leq 6$ & any
\end{tabular}

Patients with higher risk cancers were often selected for combined therapy with neoadjuvant ADT and radiation, but there was no formal policy governing this until 1997. Additionally, because of waiting lists in the early 1990's men with lower risk cancers were also given ADT. Prior to 1997, ADT was delivered by a combination of low-dose stilboestrol $(0.1 \mathrm{mg})$ and cyproterone acetate $(50 \mathrm{mg})$, which has been shown to provide castrate levels of testosterone [7], subsequently LHRH agonist injections (with initial anti-androgen to suppress any androgen flare) were used. Total androgen blockade was not the institutional policy and was used in less than $5 \%$ of patients. ADT use was mainly neo-adjuvant until 1997. In 1997, when data was presented showing a benefit from extended adjuvant ADT in high risk patients [8], the BCCA published guidelines [9] and thereafter adjuvant ADT was added to our prior neoadjuvant practice, for an increased overall duration of ADT.

Generally, patients were seen every 6 months for 3 years, then annually for 3 years, then every 2 years. At each visit clinical examination, PSA and testosterone assays, and toxicity were scored. Follow-up is timed from the completion of radiation therapy. Data for all patients was entered prospectively into the database. Additional PSA results from other sources, such as general practice requests, were also incorporated into the database. Institutional ethics review boards approval was obtained for this study.

The standard risk stratification schema was that of the National Comprehensive Cancer Network ( NCCN) with three levels: high risk - those with either PSA > $20 \mathrm{ng} / \mathrm{mL}$ or T stage T3 or more, or Gleason score 8-10; low risk those with a PSA $<10 \mathrm{ng} / \mathrm{mL}$ and T2a or less stage, and GS 6 or less; intermediate risk - all those not high or low risk. Our five level investigational risk schema is shown in Table 1.

The primary endpoint for the study is the absence of biochemical evidence of disease (bNED), which has been shown to be an independent predictor of overall survival when radiotherapy alone has been used to treat prostate cancer [10] and is frequently used as a early end-point in studies of prostate cancer. Patients in this study were assessed for the primary endpoint of biochemical control with the "Phoenix" (lowest PSA to date $+2 \mathrm{ng} / \mathrm{mL}$ ) bNED criterion, as this is robust with or without the use of ADT, unlike the ASTRO definition which is not recommended for studies using ADT[11]. Overall survival (OS) and cause-specific survival (CSS) were obtained from direct data linkage with provincial and national death registries. Assigned deaths due to prostate cancer in the absence of known metastatic relapse, or death from another cause in the presence of known metastatic prostate cancer, were checked manually by chart review. 
Comparison of patient characteristics was made using chisquared tests for categorical data and Kruskal-Wallis tests for non-parametric tests. Time to end-point events was derived using the Kaplan-Meier technique with corresponding log-rank tests of significance. Patients were censored at the time of last clinical follow-up. $\mathrm{P}$ values of $<0.05$ were considered significant.

\section{Results}

The median follow-up was 5 years for biochemical status, (range 1 month - 11 years) and 7.7 years for survival, range 3 months - 12 years). Patient characteristics by expanded and NCCN risk groups are shown in table 2. ADT was used more frequently and for progressively longer durations in higher risk groups.

The Kaplan-Meier survival curves for biochemical control by NCCN risk groups are presented in figure 1. In the low risk group there was no difference in 5 year bNED between the patients treated with ADT and without $(92 \%$ versus $85 \%$ respectively, $p=0.33$ ). In the intermediate risk group the 5 year bNED rate was significantly higher in the patients treated with ADT at $82 \%$ compared to those treated without at $70 \%(p=0.003)$. In the high risk group there was also a difference between those treated with and without ADT, $55 \%$ versus $42 \%$ respectively $(\mathrm{p}=0.004)$.

With the expanded 5-risk grouping significant differences in bNED was seen in the high-intermediate, high and extreme risk groups but not the low and low-intermediate risk groups (figure 2 and table 3 ). For the low intermedi- ate group 5 year bNED survival was $75 \%$ with ADT and $70 \%$ without $(\mathrm{p}=0.43)$. For the high intermediate group 5 year bNED survival was $72 \%$ with ADT and $55 \%$ without ( $p=0.0025)$. There was no significant effect on cancer specific survival or overall survival for any group.

\section{Discussion}

The use of androgen deprivation therapy in combination with EBRT has increased substantially over recent years, with, for example, data from the Cancer of the Prostate Strategic Urologic Research Endeavor (CAPSURE) in the United States suggesting that the rate of use has increased from $9.8 \%$ to $74.6 \%$ between $1989-1990$ and 2000 2001 [12]. This is undoubtedly related to several large randomized clinical trials which have shown a benefit to the combined treatment. This increased usage is paralleled by substantial toxicity related to castrate physiology, with physical, psychological and sexual side effects often being detrimental to a patient's quality of life. With these issues in mind, we demonstrated that there is potential to improve the selection of patients for treatment with combined hormonal manipulation based on contemporary knowledge of outcome prognostication.

To date, accurately identifying which patients will benefit from the addition of ADT to EBRT has been difficult, due to inter-trial differences in factors such as the stage and grade of the patients, dose and volume of radiation and the duration and timing of ADT. In terms of the tumour characteristics, most early studies have focused on patients with locally advanced disease. The Radiation

Table 2: Patient characteristics from the Prostate Cohort Outcomes Initiative Database of the British Columbia Cancer Agency (BCCA) sorted by NCCN risk groups [3] and 5-level risk stratification. [6]

\begin{tabular}{|c|c|c|c|c|c|c|c|c|}
\hline & \multicolumn{5}{|c|}{ Expanded Risk Groups (Williams, Duchesne,2006) } & \multicolumn{3}{|c|}{ NCCN Risk Groups } \\
\hline & Low $(n=317)$ & $\begin{array}{l}\text { Low-intermediate } \\
\quad(\mathrm{n}=293)\end{array}$ & $\begin{array}{l}\text { Intermediate } \\
\quad(\mathrm{n}=329)\end{array}$ & High $(n=24 I)$ & $\begin{array}{l}\text { Extreme } \\
(\mathrm{n}=230)\end{array}$ & Low $(n=229)$ & $\begin{array}{l}\text { Intermediate } \\
(\mathrm{n}=497)\end{array}$ & High $(n=677)$ \\
\hline \multicolumn{9}{|l|}{ PSA } \\
\hline Median & 4.9 & 10.1 & 7.7 & 15.8 & 36 & 6.1 & 9.8 & 16 \\
\hline Range & $0.2-7.5$ & $7.6-15$ & $0.3-20$ & $10.1-30$ & $20-250$ & $0.2-10$ & $0.3-20$ & $0.5-250$ \\
\hline \multicolumn{9}{|l|}{ T stage } \\
\hline TI & 70 & 77 & 46 & 22 & 23 & 116 & 82 & 40 \\
\hline $\mathrm{T} 2$ & 187 & 151 & 150 & 111 & 62 & 113 & 415 & 134 \\
\hline T3 & 56 & 58 & 126 & 97 & $12 \mid$ & 0 & 0 & 462 \\
\hline T4 & 2 & 3 & 5 & 7 & 15 & 0 & 0 & 32 \\
\hline missing & 2 & 4 & 2 & 4 & 9 & 0 & 0 & 9 \\
\hline \multicolumn{9}{|l|}{ Age } \\
\hline Median & 71 & 72 & 71 & 71 & 69 & 71 & 72 & 70 \\
\hline Age range & $46-84$ & $50-82$ & $49-86$ & $47-85$ & $48-82$ & $54-84$ & $50-86$ & $46-84$ \\
\hline \multicolumn{9}{|l|}{ EBRT dose } \\
\hline Median (Gy) & 66 & 66 & 66 & 66 & 66 & 66 & 66 & 66 \\
\hline Range (Gy) & $66-70$ & $66-70$ & $66-72$ & $66-72$ & $66-70$ & $66-70$ & $66-72$ & $66-72$ \\
\hline \multicolumn{9}{|l|}{ Gleason score } \\
\hline 6 or less & 317 & 293 & 74 & 61 & 44 & 229 & 308 & 246 \\
\hline 7 & 0 & 0 & 175 & 135 & 109 & 0 & 189 & 227 \\
\hline 8 or more & 0 & 0 & 80 & 45 & 77 & 0 & 0 & 205 \\
\hline $\begin{array}{l}\text { ADT rate [Neoadjuvant } \\
\text { alone, neoadjuvant-adjuvant] }\end{array}$ & $19.2 \%[72 \%, 28 \%]$ & $23.5 \%[55 \%, 45 \%]$ & $51.7 \%[39 \%, 61 \%]$ & $62.2 \%[36 \%, 64 \%]$ & $79.6 \%[41 \%, 59 \%]$ & $13.5 \%[87 \%, \mid 3 \%]$ & $25.4 \%[47 \%, 53 \%]$ & $69.7 \%[40 \%, 60 \%]$ \\
\hline $\begin{array}{l}\text { Duration of ADT } \\
\text { (neoadjuvant ADT) mean, } \\
\text { [total SD] in months) }\end{array}$ & $8.3(1.1)[6.0]$ & $10.8(1.5)[7.3]$ & I5.4 (3.2) [13.9] & I5.4 (4.0) [13.4] & $17.3(4.7)[16.7]$ & $7.0(0.8)[3.4]$ & $11.9(1.6)[12.0]$ & I6.2(4.3) [14.5] \\
\hline
\end{tabular}


Low risk, $p=$ n.s.

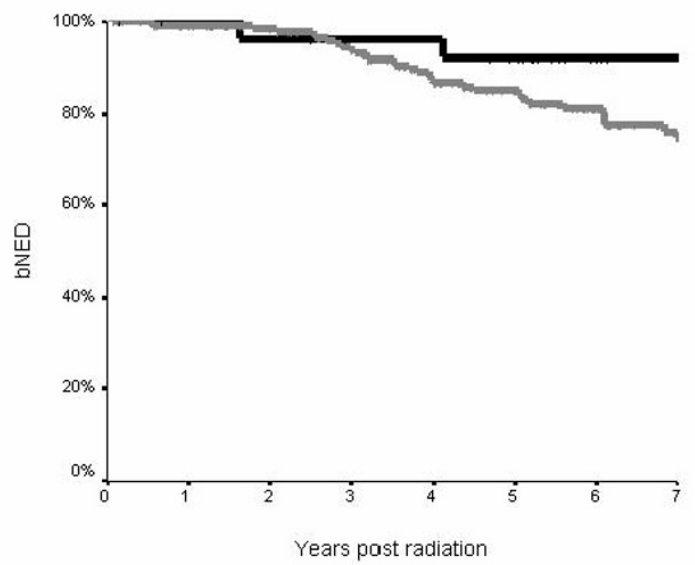

Intermediate risk, $p=0.003$

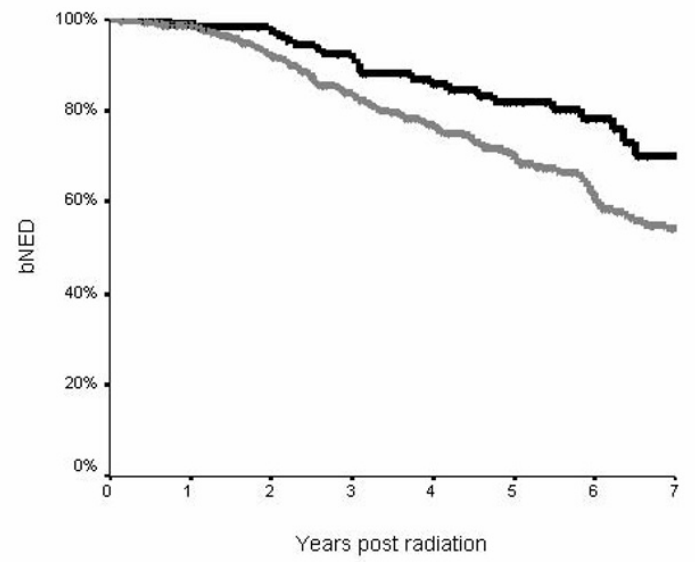

High risk, $p=0.004$

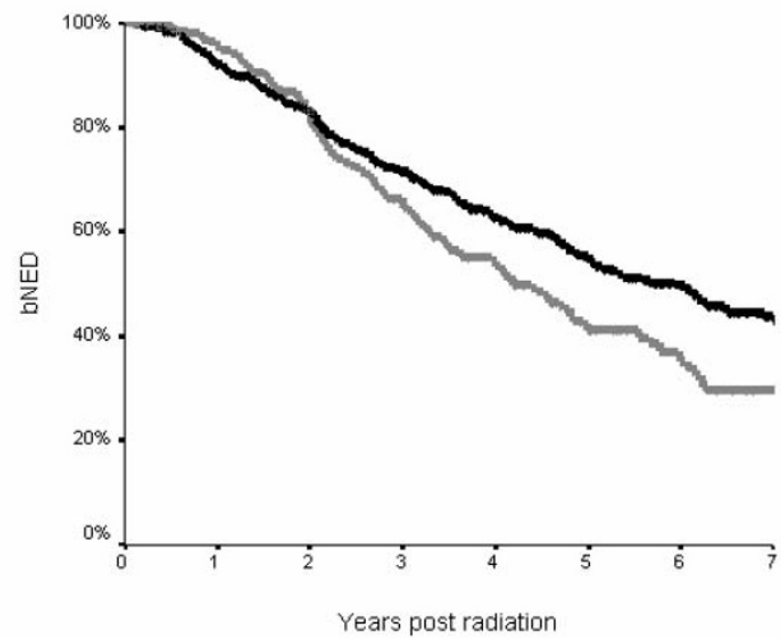

Figure I

Kaplan Meier curve for bNED (biochemical lack of evidence of disease survival) according to NCCN risk groups [3]. Black lines show combined EBRT and ADT, grey lines EBRT alone. P values refer to the log-rank test.

Table 3: 5-year biochemical lack of evidence of disease (bNED) in patients treated for prostate cancer with EBRT sorted by 5-level risk stratification, with the hazard ratio of relapse with/without ADT and corresponding $P$ values are generated from the Kaplan-Meier logrank test.

\begin{tabular}{|c|c|c|c|c|}
\hline Risk Group & With ADT & Without ADT & Hazard ratio $[95 \% \mathrm{Cl}]$ & $P$ value \\
\hline Low & $84 \%$ & $86 \%$ & $1.8[0.2-16.6]$ & n.s \\
\hline Low intermediate & $75 \%$ & $70 \%$ & I.I $[0.56-2.1]$ & n.s \\
\hline High intermediate & $72 \%$ & $55 \%$ & $0.57[0.4-0.83]$ & $P=0.0029$ \\
\hline High & $64 \%$ & $36 \%$ & $0.47[0.33-0.69]$ & $P=0.0001$ \\
\hline Extreme & $43 \%$ & $21 \%$ & $0.61[0.42-0.89]$ & $P=0.01$ \\
\hline
\end{tabular}


Low risk, $p=n s$

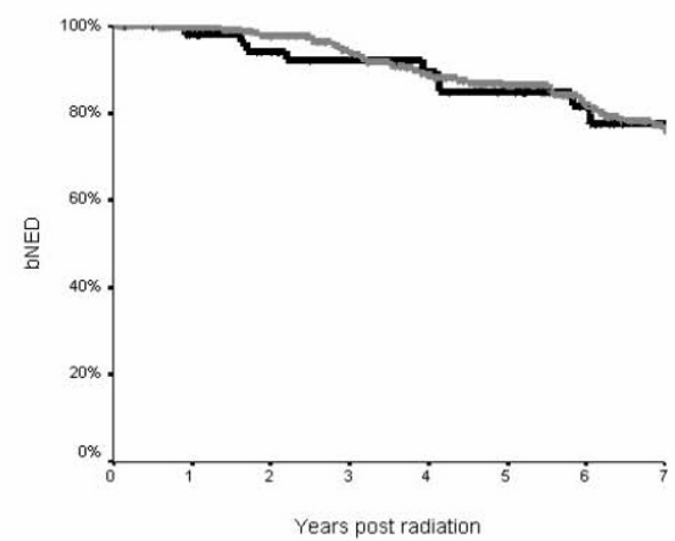

High-Intermediate risk, $p=0.0029$

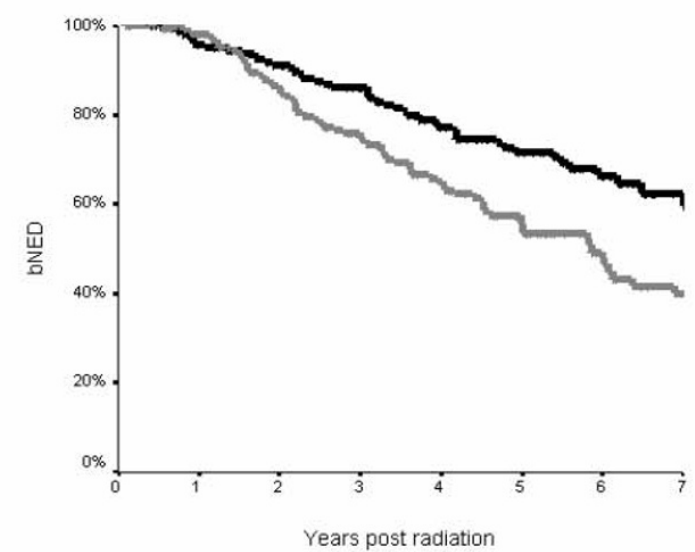

Extreme risk $p=0.01$

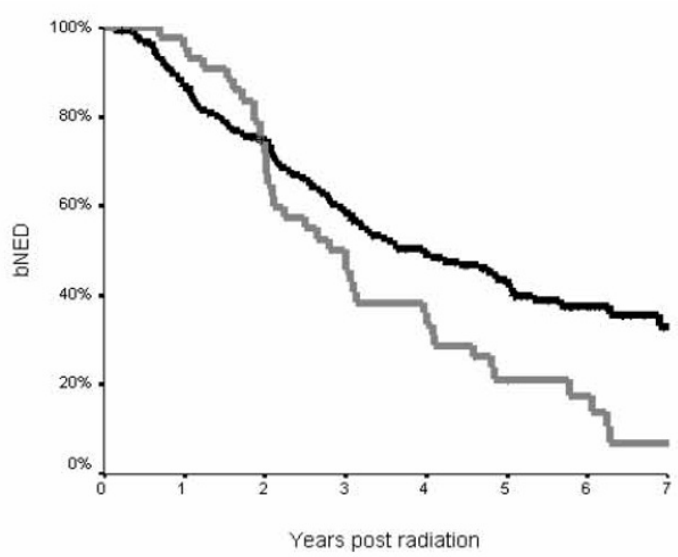

Low-intermediate risk, $p=n s$

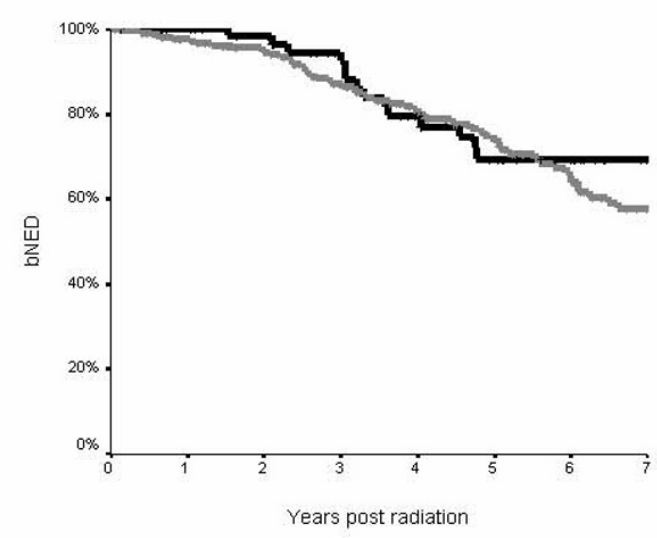

High risk, $p=0.0001$

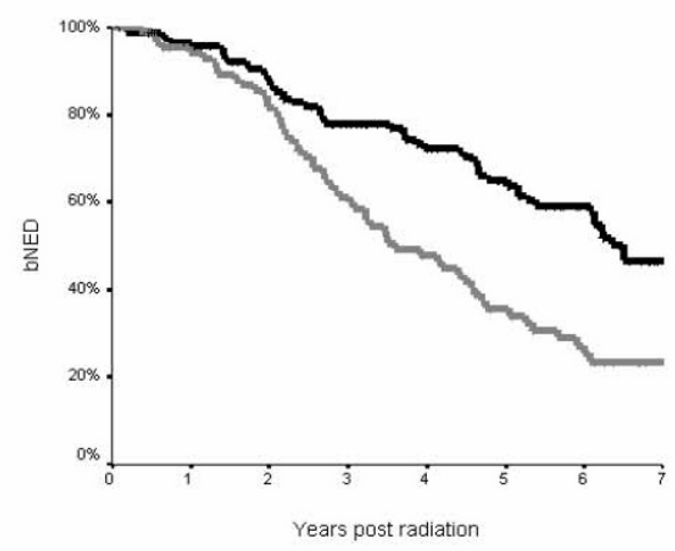

Figure 2

Kaplan Meier curve for bNED (biochemical lack of evidence of disease survival) according to expanded risk group [6]. 5-year bNED rates and hazard ratios are in Table 3. Black lines show combined EBRT and ADT, grey lines EBRT alone. $P$ values refer to the log-rank test. 
Therapy Oncology Group (RTOG) 86-10 study [13] randomized patients with bulky primary cancers to either 2 months neoadjuvant and 2 months concurrent ADT with EBRT or EBRT alone. There was improved bNED and local control at 8 years with the addition of ADT, and the subgroup with Gleason score 2-6 cancers (many notably lacked baseline PSA levels) had a statistically improved overall survival at $70 \%$ compared to $52 \%$ with EBRT alone. Similarly, locally advanced (T3-4) or node positive cancers were included in EORTC 22863 [1] and RTOG 85-31 [14] trials, and randomized to have either immediate long term ADT starting at EBRT completion, or observation with delayed ADT. Overall survival was improved from $62 \%$ to $78 \%$ at five years in the EORTC trial (p = $0.0002)$, and improved in the Gleason score 8-10 subset of the RTOG trial. The Trans-Tasman Radiation Oncology Group (TROG) 96.01 trial of locally advanced prostate cancer [2] showed a benefit of adding ADT in terms of local failure, bNED, disease free survival and freedom from salvage treatment for both 3 and 6 months of neoadjuvant ADT.

Possibly more relevant to contemporary cohorts, the trial by D'Amico et al [15] randomized 206 patients with T1b - T2b tumors with a Gleason score greater than 6 to either EBRT (70 Gy) alone or EBRT with 6 months ADT. After an average follow-up of 4.5 years the estimated overall survival at 5 years was $88 \%$ for the combined treatment arm compared to $78 \%$ for radiotherapy alone $(p=0.04)$. There were only 6 prostate-cancer deaths in this trial, and given the small patient numbers the results should be treated with caution until replicated. Overall however, the general evidence appears to be in favour of the addition of ADT to EBRT in many prostate cancers, and a variety of ADT durations have resulted in significant gains.

Logically, the duration of ADT has since become the focus in a number of studies. A Canadian study which included T1c-T4 tumors, compared 3 and 8 months neo-adjuvant ADT and showed improvements in cause-specific survival in a high risk sub-set with longer ADT durations, according to a recent oral update of a prior publication [16]. The RTOG 92-02 study looked at more prolonged ADT, comparing 4 months with 24 months. This showed improvements in local control, bNED, cause-specific survival and freedom from distant metastases with prolonged ADT but an overall survival benefit was only seen for those with Gleason score $8-10$. This benefit, curiously, was only seen in those with community-generated pathology reports, and was no longer present after central review[17]. TROG 96.01 also suggested cancer specific survival was also improved with 6 months ADT rather than zero or three months [2]. A further large TROG study ("RADAR") comparing 6 with 18 months ADT has finished accrual. The net result of these mixed studies is that
ADT is used almost universally in high risk cancers, and also for intermediate risk in many centres. Using these standard NCCN criteria our data further reinforces these findings. Additionally, we suggest that by using an alternative risk stratification, the bNED benefit of additional ADT is confined to the high-intermediate, high and extreme risk groups.

No significant effect on cancer specific or overall survival was able to be demonstrated. This may be because the follow-up is too short and/or it may reflect the relatively high short-term efficacy of salvage ADT. Another possible explanation could be that the ADT duration for the high and extreme risk groups was insufficient. Prolonging the duration of ADT appears to benefit patients with a higher chance of relapse following EBRT [18,19]. A previous analysis from our institution also demonstrated an advantage to prolonged, rather than shorter ADT duration (6 months versus 12 versus 24 months) in patients with localized disease and a PSA above 20 [20]. In our current study however, the mean durations of ADT for the high and extreme risk groups were 15 months and 17 months respectively and the median duration of ADT treatment was only 11 months in both groups. However these criticisms do not affect our main conclusions, in that a bNED benefit is a necessary precursor to a survival benefit, and no bNED benefit was seen in the low-intermediate group.

The study presented here used prospectively collected data but a valid criticism of the comparison made is that it is not a randomized trial. There was likely case selection between those patients who received ADT and those who did not. A further issue is that the doses of radiation that were used, while typical for the era, are below contemporary levels. Whether or not dose-escalation beyond the doses used in the era of this study (66-70 Gy) would obviate any benefit of ADT in higher risk cancers is currently unknown, although subject to ongoing randomized trials. Improved bNED rates seen with escalated doses of radiation would suggest that the benefit might be less than that achieved with ADT. For example the absolute improvement in Phoenix bNED with 78 Gy versus 68 Gy in the Dutch randomized trial was $6 \%$ [21], which is substantially less than that observed from additional use of ADT in the present study for higher risk cancers where the bNED improvement was $18-28 \%$ depending on risk. Possibly, according to risk category, the strategies of dose escalation and ADT will work in different ways, and it would be logical to suppose that lower risk cancers have more to gain form dose escalation, and higher risk cancers from both ADT as well as dose escalation. Furthermore, previous trials of prolonged ADT in very advanced cancers have been criticized because they do not have an ADTonly arm. The National Cancer Institute of Canada PR3/ Medical Research Council (UK) study, which randomized 
patients with locally advanced disease between ADT and ADT + EBRT, is yet to report, but should address this criticism.

ADT often causes significant side effects. During therapy, patients can suffer hot flashes, weight gain, gynaecomastia, impotence, loss of libido, fatigue and depression $[22,23]$. Longer-term side effects include a significant loss of bone mineral density after a year of ADT [24] and increased fracture rates with more prolonged durations of ADT[25]. A recently published study using the SEER database has demonstrated a significantly increased risk of developing incident diabetes, coronary heart disease, myocardial infarction and sudden cardiac death in men with prostate cancer who received ADT, when compared with those who did not [26] Even short durations of LHRH agonist therapy (1-4 months) were shown to carry increased risk of incident diabetes and coronary heart disease. D'Amico etc [27] has also shown increased fatal MI rates in those aged $>65$ years, with even short term ADT. There may also be adverse cognitive effects after 6 months of ADT [28]. Considering all these potential morbidities, ADT should be reserved for those with the highest chance of net benefit.

Overall, trials looking at the combination of ADT and EBRT have shown the greatest benefits for patients with locally advanced or high-grade tumors. This is reflected by our practice, in that higher risk patients were more likely to receive ADT. This was also the case in another retrospective review published recently [29]. The benefit of ADT in lower risk groups remains more controversial, and an improvement in bNED was not demonstrated in our dataset for low and low-intermediate risk patients. Therefore, these men (comprising 43\%, of our cohort) may be safely spared the additional toxicity of ADT without compromising tumour control.

\section{Conclusion}

This analysis divides the intermediate risk patients with localized prostate cancer and identifies the sub-groups who do, and do not obtain a bNED benefit from ADT. There appears to be no advantage to ADT where the Gleason score is 6 or less and PSA is 15 or less. ADT provides a bNED benefit in patients treated to standard dose radiation with Gleason 6 disease with a PSA greater than 15 or where the Gleason score is higher than 6 .

\section{Competing interests}

The authors declare that they have no competing interests.

\section{Authors' contributions}

MB participated in the design of the study, checked data integrity, carried out the analyses and drafted the manuscript. SGW developed the new risk group system, partici- pated in the design of the study and drafts of the manuscript. TP conceived of the study, co-developed the prospective data sources, participated in the design of the study and manuscript drafts. All authors have read and approved the final manuscript.

\section{Acknowledgements}

The BCCA Prostate Cohort Outcomes Initiative receives an unrestricted educational grant from Abbott Labs Ltd. Physicians contributing significantly to the BCCA Prostate Cohort Outcomes Initiative: Alex Agranovich, M.D., Eric Berthelet, M.D., F.R.C.P.C., Graeme Duncan, M.D., F.R.C.P.C., Mira Keyes, M.D., F.R.C.P.C., Charmaine Kim-Sing, M.B., F.R.C.P.C., Ed Kostaschuk, MD., Winkle Kwan, M.B.B.S., F.R.C.P.C., Mitchell Liu, M.D.C.M., F.R.C.P.C., Michael McKenzie, M.D., F.R.C.P.C., W James Morris M.D., F.R.C.P.C., Milton Po, M.D., F.R.C.P.C., and Jane Wilson, M.D., F.R.C.P.C.

\section{References}

I. Bolla M, Collette L, Blank L, Warde P, Dubois JB, Mirimanoff RO, Storme G, Bernier J, Kuten A, Sternberg C, Mattelaer J, Lopez Torecilla J, Pfeffer JR, Lino Cutajar C, Zurlo A, Pierart M: Long-term results with immediate androgen suppression and external irradiation in patients with locally advanced prostate cancer (an EORTC study): a phase III randomised trial. Lancet 2002, 360(9327): 103-106.

2. Denham JW, Steigler A, Lamb DS, Joseph D, Mameghan H, Turner S, Matthews J, Franklin I, Atkinson C, North J, Poulsen M, Christie D, Spry NA, Tai KH, Wynne C, Duchesne G, Kovacev O, D'Este C: Short-term androgen deprivation and radiotherapy for locally advanced prostate cancer: results from the TransTasman Radiation Oncology Group $\mathbf{9 6 . 0 1}$ randomised controlled trial. Lancet Oncol 2005, 6( I I ):84 I-850.

3. National_Comprehensive_Cancer_Network: NCCN Clinical Practice Guidelines in Oncology. 2007.

4. D'Amico AV, Schultz D, Loffredo M, Dugal R, Hurwitz M, Kaplan I, Beard CJ, Renshaw AA, Kantoff PW: Biochemical outcome following external beam radiation therapy with or without androgen suppression therapy for clinically localized prostate cancer. Jama 2000, 284( I0): I 280- I 283.

5. Williams SG, Millar JL, Dally MJ, Sia S, Miles W, Duchesne GM: What defines intermediate-risk prostate cancer? Variability in published prognostic models. Int J Radiat Oncol Biol Phys 2004, 58(I): I I-18.

6. Williams SG, Duchesne GM, Gogna NK, Millar JL, Pickles T, Pratt GR, Turner S: An international multicenter study evaluating the impact of an alternative biochemical failure definition on the judgment of prostate cancer risk. Int J Radiat Oncol Biol Phys 2006, 65(2):351-357.

7. Goldenberg SL, Bruchovsky N, Gleave ME, Sullivan LD: Low-dose cyproterone acetate plus mini-dose diethylstilbestrol--a protocol for reversible medical castration. Urology 1996, 47(6):882-884.

8. Bolla M, Gonzalez D, Warde P, Dubois JB, Mirimanoff RO, Storme G, Bernier J, Kuten A, Sternberg C, Gil T, Collette L, Pierart M: Improved survival in patients with locally advanced prostate cancer treated with radiotherapy and goserelin. $N$ Engl J Med 1997, 337(5):295-300.

9. BC_Cancer_Agency: Androgen Deprivation Guidelines. [http/ www.bccancer.bc.ca/HPI/CancerManagementGuidelines/Genitouri nary/Prostate/Management/HighRisk.htm]

10. Kwan W, Pickles T, Duncan G, Liu M, Agranovich A, Berthelet E, Keyes M, Kim-Sing C, Morris WJ, Paltiel C: PSA failure and the risk of death in prostate cancer patients treated with radiotherapy. Int J Radiat Oncol Biol Phys 2004, 60(4): I 040- 1046.

II. Roach M 3rd, Hanks G, Thames H Jr., Schellhammer P, Shipley WU, Sokol GH, Sandler H: Defining biochemical failure following radiotherapy with or without hormonal therapy in men with clinically localized prostate cancer: Recommendations of the RTOG-ASTRO Phoenix Consensus Conference. Int J Radiat Oncol Biol Phys 2006, 65(4):965-974.

12. Cooperberg MR, Broering JM, Litwin MS, Lubeck DP, Mehta SS, Henning JM, Carroll PR: The contemporary management of pros- 
tate cancer in the United States: lessons from the cancer of the prostate strategic urologic research endeavor (CapSURE), a national disease registry. J Urol 2004, | 7 |(4): |393-|40|.

13. Pilepich MV, Winter K, John MJ, Mesic JB, Sause W, Rubin P, Lawton $C$, Machtay M, Grignon D: Phase III radiation therapy oncology group (RTOG) trial 86-10 of androgen deprivation adjuvant to definitive radiotherapy in locally advanced carcinoma of the prostate. Int J Radiat Oncol Biol Phys 200I, 50(5): I243-I 252.

14. Lawton CA, Winter K, Murray K, Machtay M, Mesic JB, Hanks GE, Coughlin CT, Pilepich MV: Updated results of the phase III Radiation Therapy Oncology Group (RTOG) trial 85-3 I evaluating the potential benefit of androgen suppression following standard radiation therapy for unfavorable prognosis carcinoma of the prostate. Int J Radiat Oncol Biol Phys 200I, 49(4):937-946.

15. D'Amico AV, Manola J, Loffredo M, Renshaw AA, DellaCroce A, Kantoff PW: 6-month androgen suppression plus radiation therapy vs radiation therapy alone for patients with clinically localized prostate cancer: a randomized controlled trial. Jama 2004, 292(7):82I-827.

16. Crook J, Ludgate C, Malone S, Lim J, Perry G, Eapen L, Bowen J, Robertson S, Lockwood G: Report of a multicenter Canadian phase III randomized trial of 3 months vs. 8 months neoadjuvant androgen deprivation before standard-dose radiotherapy for clinically localized prostate cancer. Int J Radiat Oncol Biol Phys 2004, 60(I):15-23.

17. Hanks GE, Pajak TF, Porter A, Grignon D, Brereton H, Venkatesan V, Horwitz EM, Lawton C, Rosenthal SA, Sandler HM, Shipley WU: Phase III trial of long-term adjuvant androgen deprivation after neoadjuvant hormonal cytoreduction and radiotherapy in locally advanced carcinoma of the prostate: the Radiation Therapy Oncology Group Protocol 92-02. J Clin Oncol 2003, 2I(2I):3972-3978.

18. Horwitz EM, Winter K, Hanks GE, Lawton CA, Russell AH, Machtay M: Subset analysis of RTOG 85-3I and 86-10 indicates an advantage for long-term vs. short-term adjuvant hormones for patients with locally advanced nonmetastatic prostate cancer treated with radiation therapy. Int J Radiat Oncol Biol Phys 200I, 49(4):947-956

19. Bolla M, VanTienhoven G, de Reijke T, van der Bergh A, van der meijden A, Poortmans P, Gez E, Kil PJ, Pierart M, Collette L, EORTC Radiation Oncology and Genito-Urinary TractCancer Groups: Concomitant and adjuvant androgen deprivation therapy with external beam irradiation for locally advanced prostate cancer: 6 months versus 3 years ADT, Results of the randomised EORTC phase 3 trial 2296I. Journal of Clinical Oncology 2007, ASCO Meeting Abstracts Jun 20 2007: 5014.:

20. Berthelet E, Pickles T, Truong PT, Liu M, Pai HH, Kwan WB, Lim JT: What is the optimal duration of androgen deprivation therapy in prostate cancer patients presenting with prostatespecific antigen levels > $20 \mathrm{ng} / \mathrm{ml}$ ? Can J Urol 2007, I4(4):3621-3627.

21. Peeters ST, Heemsbergen WD, Koper PC, van Putten WL, Slot A, Dielwart MF, Bonfrer JM, Incrocci L, Lebesque JV: Dose-response in radiotherapy for localized prostate cancer: results of the Dutch multicenter randomized phase III trial comparing 68 Gy of radiotherapy with 78 Gy. J Clin Oncol 2006, 24(13): 1990-1996.

22. Sharifi N, Gulley JL, Dahut WL: Androgen deprivation therapy for prostate cancer. Jama 2005, 294(2):238-244.

23. Holzbeierlein JM, McLaughlin MD, Thrasher JB: Complications of androgen deprivation therapy for prostate cancer. Curr Opin Urol 2004, I 4(3): I77-183.

24. Mittan D, Lee S, Miller E, Perez RC, Basler JW, Bruder JM: Bone loss following hypogonadism in men with prostate cancer treated with GnRH analogs. J Clin Endocrinol Metab 2002, 87(8):3656-366I.

25. Shahinian VB, Kuo YF, Freeman JL, Goodwin JS: Risk of fracture after androgen deprivation for prostate cancer. N Engl J Med 2005, 352(2): 154-164.

26. Keating NL, O'Malley AJ, Smith MR: Diabetes and cardiovascular disease during androgen deprivation therapy for prostate cancer. J Clin Oncol 2006, 24(27):4448-4456.

27. D'Amico AV, Denham JW, Crook J, Chen MH, Goldhaber SZ, Lamb DS, Joseph D, Tai KH, Malone S, Ludgate C, Steigler A, Kantoff PW:
Influence of androgen suppression therapy for prostate cancer on the frequency and timing of fatal myocardial infarctions. J Clin Oncol 2007, 25( 17):2420-2425.

28. Green HJ, Pakenham KI, Headley BC, Yaxley J, Nicol DL, Mactaggart PN, Swanson C, Watson RB, Gardiner RA: Altered cognitive function in men treated for prostate cancer with luteinizing hormone-releasing hormone analogues and cyproterone acetate: a randomized controlled trial. BJU Int 2002, 90(4):427-432.

29. Zeliadt SB, Potosky AL, Penson DF, Etzioni R: Survival benefit associated with adjuvant androgen deprivation therapy combined with radiotherapy for high- and low-risk patients with nonmetastatic prostate cancer. Int J Radiat Oncol Biol Phys 2006, 66(2):395-402.
Publish with Biomed Central and every scientist can read your work free of charge

"BioMed Central will be the most significant development for disseminating the results of biomedical research in our lifetime. "

Sir Paul Nurse, Cancer Research UK

Your research papers will be:

- available free of charge to the entire biomedical community

- peer reviewed and published immediately upon acceptance

- cited in PubMed and archived on PubMed Central

- yours - you keep the copyright

Submit your manuscript here:

http://www.biomedcentral.com/info/publishing_adv.asp 